\title{
The impact of health programmes to prevent vertical transmission of HIV. Advances, emerging health challenges and research priorities for children exposed to or living with HIV: Perspectives from South Africa
}

\author{
A Goga, ${ }^{1,2,3} \mathrm{PhD}$; A Slogrove, ${ }^{4} \mathrm{PhD}$; C J Wedderburn, ${ }^{5,6} \mathrm{MSc}$; U Feucht,${ }^{2,7-9} \mathrm{PhD}$; J Wessels, ${ }^{8} \mathrm{MPH} ; \mathrm{V}$ Ramokolo, ${ }^{1} \mathrm{PhD} ;$ A Bhana, ${ }^{1,10} \mathrm{PhD}$; \\ N du Plessis, ${ }^{2} \mathrm{PhD}$; R J Green, ${ }^{2} \mathrm{DSc}$, Y Pillay, ${ }^{11} \mathrm{PhD}$; G Sherman, ${ }^{12,13} \mathrm{PhD}$ \\ ${ }^{1}$ Health Systems Research Unit, South African Research Council, Cape Town, South Africa \\ ${ }^{2}$ Department of Paediatrics and Child Health, Faculty of Health Sciences, University of Pretoria, South Africa \\ ${ }^{3}$ HIV Prevention Research Unit, South African Medical Research Council, Cape Town, South Africa \\ ${ }^{4}$ Department of Paediatrics and Child Health, Faculty of Medicine and Health Sciences, Stellenbosch University, Cape Town, South Africa \\ ${ }^{5}$ Department of Paediatrics and Child Health, Red Cross War Memorial Children's Hospital and University of Cape Town, South Africa \\ ${ }^{6}$ Department of Clinical Research, London School of Hygiene \& Tropical Medicine, London, UK \\ 7 Tshwane District Health Services, Gauteng Department of Health, Pretoria, South Africa \\ ${ }^{8}$ Research Centre for Maternal, Fetal, Newborn and Child Health Care Strategies, Faculty of Health Sciences, University of Pretoria, South Africa \\ ${ }^{9}$ Maternal and Infant Health Care Strategies Research Unit, South African Medical Research Council, Pretoria, South Africa \\ ${ }^{10}$ Centre for Rural Health, School of Nursing and Public Health, University of KwaZulu-Natal, Durban, South Africa \\ ${ }^{11}$ National Department of Health, Pretoria, South Africa \\ ${ }^{12}$ Department of Paediatrics and Child Health, Faculty of Health Sciences, University of the Witwatersrand, Johannesburg, South Africa \\ ${ }^{13}$ Centre for HIV \& STI, National Institute for Communicable Diseases, National Health Laboratory Services, Johannesburg, South Africa
}

Corresponding author: A Goga (ameena.goga@mrc.ac.za)

Over the past three decades, tremendous global progress in preventing and treating paediatric HIV infection has been achieved. This paper highlights the emerging health challenges of HIV-exposed uninfected (HEU) children and the ageing population of children living with HIV (CLHIV), summarises programmatic opportunities for care, and highlights currently conducted research and remaining research priorities in high HIV-prevalence settings such as South Africa. Emerging health challenges amongst HEU children and CLHIV include preterm delivery, suboptimal growth, neurodevelopmental delay, mental health challenges, infectious disease morbidity and mortality, and acute and chronic respiratory illnesses including tuberculosis, pneumonia, bronchiectasis and lymphocytic interstitial pneumonitis. CLHIV and HEU children require three different categories of care: $(i)$ optimal routine child health services applicable to all children; (ii) routine care currently provided to all HEU children and CLHIV, such as HIV testing or viral load monitoring, respectively, and (iii) additional care for CLHIV and HEU children who may have growth, neurodevelopmental, behavioural, cognitive or other deficits such as chronic lung disease, and require varying degrees of specialised care. However, the translation thereof into practice has been hampered by various systemic challenges, including shortages of trained healthcare staff, suboptimal use of the patient-held child's Road to Health book for screening and referral purposes, inadequate numbers and distribution of therapeutic staff, and shortages of assistive/diagnostic devices, where required. Additionally, in low-middle-income high HIV-prevalence settings, there is a lack of evidence-based solutions/models of care to optimise health amongst HEU and CLHIV. Current research priorities include understanding the mechanisms of preterm birth in women living with HIV to optimise preventive interventions; establishing pregnancy pharmacovigilance systems to understand the short-, medium- and long-term impact of in utero ART and HIV exposure; understanding the role of preconception maternal ART on HEU child infectious morbidity and long-term growth and neurodevelopmental trajectories in HEU children and CLHIV, understanding mental health outcomes and support required in HEU children and CLHIV through childhood and adolescence; monitoring HEU child morbidity and mortality compared with HIV-unexposed children; monitoring outcomes of CLHIV who initiated ART very early in life, sometimes with suboptimal ART regimens owing to medication formulation and registration issues; and testing sustainable models of care for HEU children and CLHIV including later reproductive care and support.

S Afr Med J 2019;109(11 Suppl 1):77-82. https://doi.org/10.7196/SAMJ.2019.v109i11b.14292

Over the past three decades, tremendous global progress in preventing and treating paediatric HIV infection has been achieved. ${ }^{[1]}$ In 2009, it was projected that $95 \%$ coverage of dual therapy to prevent vertical transmission of HIV with appropriate feeding choices could save 37200 children's lives in 2015, using 2008 data as a baseline. ${ }^{[2]}$ Although life-long triple antiretroviral therapy (ART) for pregnant and breastfeeding women has not been the panacea for eliminating vertical HIV transmission, ${ }^{[1]}$ it has certainly brought this goal significantly closer $^{[1,3]}$ Finally, we are seeing a shift to fewer perinatally acquired HIV infections; however, concomitantly, owing to sustained high antenatal HIV prevalence, the population of HIV-exposed uninfected (HEU) children is steadily increasing. Additionally we are seeing an ageing cohort of children living with HIV (CLHIV) ${ }^{[4]}$ 
Researchers associated with the South African Medical Research Council (SAMRC) have conducted pivotal research, firstly to prevent MTCT and subsequently to attain EMTCT. The current SAMRC president, Glenda Gray, played a key role in the first multi-country PErinatal TRAnsmission (PETRA) tiral to investigate the efficacy of three short-course maternal and infant antiretroviral drug regimens to prevent MTCT. ${ }^{[5]}$ The PETRA trial demonstrated that short-course, dual maternal antiretroviral (ARV) prophylaxis (from 36 weeks' gestation until 7 days postpartum) significantly reduced MTCT by 4 - 8 weeks postdelivery (63\% efficacy), but had no significant 18-month benefits. This trial was followed by pivotal infant ARV post exposure prophylaxis research to obviate MTCT in women with no prior ART exposure. ${ }^{[6]}$ Once South Africa (SA) adopted a policy to provide single-dose nevirapine to prevent vertical HIV transmission (PMTCT) in 18 pilot sites, the SAMRC was a key partner in evaluating the effectiveness of this policy: research in three purposively selected sites (Paarl, Rietvlei and Umlazi) demonstrated $8.6 \%, 13.7 \%$ and $11.9 \%$ MTCT at $3-4$ weeks, ${ }^{[7]}$ and $84 \%, 65 \%$ and $74 \%$ HIV-free survival at 9 months, ${ }^{[8]}$ respectively, and poor infant feeding practices amongst HIV-negative and -positive mothers in the context of PMTCT. ${ }^{[9]}$ After 8 years of national PMTCT scale-up, the SAMRC led 3 nationally representative evaluations in 2010, $2011-2012$ and $2012-2013,^{[10,11]}$ and 1 prospective observational cohort study ${ }^{[12]}$ to measure 6-week and 18-month PMTCT effectiveness, respectively. These demonstrated a reduction in national 6-week MTCT to $3.5 \%$ with dual therapy (2008 PMTCT guidelines) and 2.6\% with PMTCT Option A, and $4.3 \%$ at 18 months, with $6.3 \% 18$-month MTCT or death. Since these surveys, a SAMRC-National Institute of Communicable Diseases (NICD) collaboration has established that national laboratory data provide valid estimates of early national MTCT. ${ }^{[13]}$ With the adoption of PMTCT Option B+ (lifelong triple ART) for pregnant and breastfeeding women in 2015, and the release of global criteria to validate EMTCT, national short- and longterm PMTCT effectiveness needs to be monitored. ${ }^{[3,14,15]}$ Despite a reduction in MTCT at national, provincial and district levels, SAMRC and partners report differential MTCT across provinces and districts, highlighting the gap between current case rates and the global target of 50 or fewer new paediatric HIV infections per 100000 livebirths. ${ }^{[16]}$ In 2019, the SAMRC led an analysis paper on whether elimination of MTCT is achievable in high HIV-prevalence settings, highlighting how maternal HIV prevalence drives the paediatric HIV case rate. ${ }^{[17]}$

By 2019, SA has stabilised antenatal HIV prevalence and been hugely successful in reducing MTCT. ${ }^{[16,18,19]}$ Expressed as a percentage of all births to women living with HIV (WLHIV), modelling demonstrates that total MTCT (perinatal and postnatal HIV transmission to end of breastfeeding) decreased from 31.2\% (95\% confidence interval (CI) 28.7 - 34.3) in mid-2004/05, to $5.2 \%$ (95\% CI 4.5 - 6.1) in mid-2016/17 (Thembisa model version 4.1). Overall, perinatal HIV transmission decreased from $18.7 \%$ (95\% CI 18.2 - 19.2) in mid2004/05 to $1.3 \%$ (95\% CI 1.1 - 1.5) in mid-2016/17. Perinatal HIV transmission amongst mothers with known HIV infection was $0.84 \%$ (95\% CI $0.78-0.90)$ in mid-2016/17. Consequently, given the stable antenatal HIV prevalence and reduction in MTCT, the number of HEU children aged 0 - 14 years increased from 1.3 million in 2004 to 3.2 million in 2017 - this is the highest number within any country globally, representing $21.5 \%$ of the global HEU child population. ${ }^{[4]}$

This paper highlights the emerging health challenges of HEU children and the ageing population of CLHIV, summarises programmatic opportunities for care, and highlights both current research underway and remaining research priorities in high HIVprevalence settings, such as SA.

\section{What are the emerging health challenges faced by HEU children and CLHIV?}

\section{Newborn outcomes}

The newborn period presents a crucial opportunity for preventing perinatal and postnatal HIV transmission, through identification of all infants born to WLHIV and stratification of mother-infant pairs at high or low risk of HIV transmission with appropriate postnatal prophylaxis, depending on risk. Furthermore, all HIV-exposed newborns should receive HIV testing with a polymerase chain reaction (PCR) test to identify in utero HIV-infected infants as early as possible, as immediate initiation of infant ART improves survival and long-term outcomes. HIV-exposed newborns, both HIVinfected and HIV-uninfected, are more likely to start life as preterm infants, with lower birthweight and/or as small-for-gestational age, than their HIV-unexposed counterparts. ${ }^{[20]}$ This effect differs by maternal ART regimen and timing of treatment initiation, although observed associations related to timing may be subject to selection bias. ${ }^{[21]}$ Several studies have reported higher rates of preterm birth in infants born to women on ritonavir-boosted protease inhibitorcontaining ART than other regimens. ${ }^{[22-24]}$ These adverse birth outcomes persist even when pregnant WLHIV receive universal ART with current efavirenz or dolutegravir-based regimens, ${ }^{[25,26]}$ and are probably associated with conception on ART, particularly in low- and middle-income countries. ${ }^{[27]}$ These adverse birth outcomes are poorly understood but likely to influence HEU child outcomes beyond birth. Additionally, the potential for teratogenic effects of in utero drug exposures was recently highlighted by the preliminary signal in Botswana of a possible association between an increased risk for fetal neural tube defects and dolutegravir use at time of conception. ${ }^{[28]}$ While more definitive data are awaited, this finding highlights the critical need for pregnancy pharmacovigilance systems in low- and middle-income countries, particularly those with a high HIV burden, such as SA. ${ }^{[29]}$

\section{Growth}

The synergistic interactions between infection, immunity and childhood malnutrition are well established. ${ }^{[30]}$ Indeed, malnutrition is sometimes referred to as an immunodeficiency syndrome as it is a composite outcome of several inflammatory and metabolic pathways. ${ }^{[31]}$ Infectious diseases such as HIV infection exacerbate undernutrition. In a prospective cohort study conducted prior to the availability of maternal ART, Ramokolo and colleagues reported worse postnatal growth velocity in SA CLHIV than HIV-unexposed and HEU children. ${ }^{[32]}$ Consistent with other studies, ${ }^{[33]}$ no significant differences in growth were observed between HIV-unexposed and HEU children. However, recent data show poorer early growth of HEU children, and notably suboptimal growth and failure to catch up postnatally in HEU infants born small for gestational age compared with HIV-unexposed infants. ${ }^{[34]}$ This observation suggests a fetal origin to later poor HEU child outcomes that requires further interrogation. ${ }^{[35]}$ Data also show that maternal HIV infection is associated with several adverse birth outcomes such as low birthweight, preterm birth, small-for-gestational age and stillbirth. ${ }^{[36-38]}$ Additionally, HEU children in the era of Option $\mathrm{B}+$ have poorer height growth compared with weight, and therefore higher BMI, and obesity remain a concern in adolescence and adulthood (du Plessis - in press). The mechanisms behind these observations remain unclear. Some speculate that immune 
dysfunction induced by HIV exposure may partly explain the growth faltering. ${ }^{[39]}$ There is also evidence that in utero exposure to maternal ART increases the risk of suboptimal birth outcomes, leading to poorer growth postnatally. ${ }^{[35,40]}$ As countries adopt lifelong ART for pregnant and lactating women (WHO Option B+ policy), more women with higher CD4 counts will conceive on ART. Few data are available on these women and their children. Further research is therefore required to understand the interplay between HIV and growth in the current ART era to ensure that HIV-exposed children not only survive but also thrive.

\section{Early neurodevelopment}

Neurological, developmental and behavioural problems in CLHIV have been well-established as devastating consequences of HIV infection, ranging from cognitive deficits to HIV encephalopathy, ${ }^{[41-43]}$ and these are associated with neuro-radiological findings ${ }^{[4,45]}$ Since the introduction of paediatric ART, studies have continued to document neurodevelopmental impairment. ${ }^{[46-48]}$ Furthermore, there is growing evidence that HEU children may experience early developmental impairment. Previous reviews have reported some delays in cognitive, language or motor development, ${ }^{[47,49,50]}$ although to a lesser extent than in CLHIV and mainly in low-resource settings. ${ }^{[47]}$ A recent study from SA found motor and cognitive delay, particularly associated with preterm birth, ${ }^{[51]}$ and a study from Zambia reported a later impact on school performance. ${ }^{[52]}$ However, other studies in Botswana ${ }^{[53]}$ and $\mathrm{SA}^{[54]}$ have not found substantial differences when compared with HIV-unexposed children, although some language impairment was demonstrated, which has also been seen in high-income settings ${ }^{[55]}$ Further research and follow-up of both CLHIV and HEU children in the current ART era is needed to determine the nature of the developmental delay as well as the long-term implications. In addition, investigating the mechanisms behind the impaired development is important to prevent future developmental delay, and to understand the complex combination of HIV-related variables and illness, ART exposure and side-effects, and socioeconomic factors.

\section{Later mental health and neurocognitive development}

The impact of perinatal HIV transmission is experienced well into adolescence, where HIV has historically been implicated in normative developmental challenges, including pubertal delays, ${ }^{[56]}$ continued neurodevelopmental and cognitive problems ${ }^{[5,58]}$ as well as greater social and emotional immaturity relative to same-age peers. ${ }^{[59]}$ The most common mental health problems seem to be attention deficit hyperactivity disorder, mood disorder, anxiety disorder and substance use disorder ${ }^{[60]}$ However, not all adolescents living with HIV (ALHIV) experience the same level of vulnerability, as these disorders range from $8 \%$ to $60 \%$ in untreated HIV-infected adolescents. ${ }^{[60]}$ The evidence from high-income settings suggests that CLHIV are at significant risk for mental health problems and substance use when entering adolescence. ${ }^{[61]}$ Additionally, there is limited but growing research which suggests that youth living with perinatally acquired HIV infection may be equally, if not more, vulnerable to emotional and behavioural problems than HEU youth or HIV-uninfected youth living in households affected by HIV.$^{[62,63]}$ Recent SA data warned against conflating all HIV-infected adolescents: perinatally infected adolescents were more likely to be ART adherent, retained in healthcare and treated well by clinic staff than horizontally infected adolescents who display more mental health disorders than the former. ${ }^{[64]}$ These better outcomes amongst perinatally infected adolescents are substantiated by a recent SA study which described a lower incidence of behavioural problems amongst perinatally acquired ALHIV, compared with uninfected adolescents. ${ }^{[65]}$ In addition, the loss of adult caregivers to HIV, and poverty related to loss of income due to ill health, may also compromise the family's capacity to meet the needs of ALHIV. ${ }^{[66]}$

\section{Infectious disease morbidity and mortality}

Without ART, over $50 \%$ of CLHIV would die before age 2 years. ${ }^{[67]}$ Recent studies indicate that, despite early introduction of ART, child mortality in CLHIV remains high and viral suppression is often suboptimal, with substantial implications for future health outcomes. ${ }^{[68,69]} \mathrm{HEU}$ children have also been found to have a higher mortality than HIV-unexposed children, ${ }^{[67,70]}$ particularly in the first years of life, estimated to be $\sim$ double that of HIV-unexposed children, although reports vary across countries. ${ }^{[71,72]}$ Data from SA suggest that the excess mortality in HEU children contributes substantially to total infant mortality. ${ }^{[73]}$ HEU child mortality appears to be predominantly due to infectious causes, ${ }^{[74]}$ and SA studies have shown that HEU infants have a higher incidence as well as greater risk for mortality from invasive pneumococcal disease and viral respiratory infections. ${ }^{[75,76]}$ Prolonged breastfeeding, although with overwhelming benefits for improved nutrition and reduced diarrhoeal disease morbidity, did not prevent additional respiratory morbidity and mortality in HEU children from two different urban settings (Soweto and Paarl). ${ }^{[77,78]}$ Furthermore, in studies from Botswana, cotrimoxazole prophylaxis did not reduce infectious morbidity or improve survival in HEU children in the first year of life. ${ }^{[79]}$ Promising evidence from highincome settings suggests initiation of maternal ART before pregnancy may mitigate some of this infectious morbidity risk for HEU children; however, this still requires evaluation in HIV high-burden countries. ${ }^{[80]}$

\section{Respiratory-related morbidity and mortality}

Respiratory disease including tuberculosis and viral and bacterial infections remains the primary cause of hospital admissions in CLHIV despite early ART.[68] This finding is similar for HEU children, who also have increased rates of hospitalisation and suffer from more severe and unusual infectious diseases in the early years of life, ${ }^{[81,82]}$ particularly pneumonia. ${ }^{[83]}$ Although there is considerable difficulty estimating the association between HIV and pulmonary tuberculosis (PTB) in African children, the epidemiology of childhood TB and HIV overlap considerably. ${ }^{[8,85]}$ Routine programmatic data from Cape Town prior to the paediatric ART roll-out reported a TB incidence of 1596 per $100000^{[86]}$ in CLHIV, decreasing considerably following ART access, ${ }^{[87,88]}$ and linked also to a reduction in associated hospitalisations. ${ }^{\left[{ }^{[9]}\right.}$ HIV infection associated with multidrug-resistant (MDR) paediatric TB is a challenge, and SA studies reported a paediatric HIV prevalence of 54\%, 77\% and $43 \%$ amongst children with MDR TB in Johannesburg, KwaZulu-Natal and Cape Town, Western Cape in 2008, 2009 - 2010 and 2003 - 2008 respectively. ${ }^{[90-92]}$ Amongst HEU children, data demonstrate that the incidence of TB is four times higher than in the general population. ${ }^{\left[{ }^{[8]}\right.}$ Pneumocystis pneumonia (PJP) remains an important cause of severe pneumonia in HEU children and CLHIV: a prospective intervention study conducted in Steve Biko Academic Hospital, Pretoria, SA, demonstrated co-infection between PJP and cytomegalovirus (CMV), with a $30 \%$ mortality rate. ${ }^{[93]}$ There is compelling evidence that infant ART decreases mortality from invasive pneumonia by $62.5 \%$ in SA and protects against hospitalisation and mortality from influenza. ${ }^{[84]}$ Data are needed on the impact of PMTCT Option B+ and of early infant HIV diagnosis with early infant ART initiation on childhood respiratory diseases amongst CLHIV. Chronic lung disease (CLD), including bronchiectasis and lymphocytic interstitial pneumonitis, is 
an unresolved challenge in CLHIV, despite ART. A Zimbabwean study by Ferrand et al. ${ }^{[94]}$ demonstrated that $\sim 45 \%$ of CLHIV aged 0 - 14 years receiving HIV-related care, have CLD, even at high CD4 cell counts and despite ART access. Data on acute and chronic lung disease in CLHIV in SA are sparse. Weber et al. ${ }^{[95]}$ reporting on data from 56 children aged 5 years (interquartile range $2-8$ years) demonstrated a significant association between CLD and lung hyperinflation and hyperpigmented skin lesions. Calligaro and Gray, ${ }^{[96]}$ using data from Cape Town, SA, demonstrated increased airway resistance in HIV-infected v. HIVuninfected children, and that an abnormal forced expiratory volume in one second (FEV1) was significantly correlated with high airway resistance. Like Ferrand et al., ${ }^{[94]}$ they concluded that lung involvement occurs early in CLHIV who do not access ART and that clinical and immunological correlates are poor predictors of lung involvement. More data are needed to understand these mechanisms. Additionally, data on the lung microbiome in CLHIV are limited, warranting further investigation.

\section{What health services/ programmes exist to meet the needs of HEU children and CLHIV?}

CLHIV and HEU children require three different categories of care. ${ }^{[97]}$ The first entails optimal routine child health services applicable to all children, including immunisations, growth monitoring and nutritional support, as well as diagnosis and management of acute childhood illnesses. The second category pertains to the routine care currently provided to all HEU children and CLHIV, such as HIV testing at specified intervals (for HEU children), antiretroviral prophylaxis (for HEU children) or viral load monitoring (for CLHIV). Both these aspects of care are relatively well catered for and resourced through the Integrated Management of Childhood Illnesses (IMCI) strategy and supported by the Road to Health booklet (RTHB).The concept of what constitutes 'routine care' and 'success' in terms of the PMTCT programme, however, requires a paradigm shift in SA. The current major endpoint of the PMTCT programme is a child who is HIV free. A more progressive view of success would be a child who is HIV free, thriving, and has normal neurodevelopment, for which the third category of care is also required. The third category is additional care for CLHIV and HEU children who may have growth, neurodevelopmental, behavioural cognitive or other deficits such as chronic lung disease, and require varying degrees of specialised care. The new national PMTCT guideline (2019) identifies the HEU child as being at risk for poorer outcomes and recommends vigilance to identify vulnerabilities. The RTHB, the IMCI strategy, and the School Health Programme have included screening for developmental impairments in routine care for all children, including HEU children and CLHIV. ${ }^{[98]}$ However, the translation thereof into practice has been hampered by various systemic challenges, including shortages of trained healthcare staff, suboptimal use of the patient-held RTHB for screening and referral purposes, inadequate numbers and distribution of therapeutic staff, lack of long-term follow-up of HEU children and adolescents, and shortages of assistive/diagnostic devices where required. ${ }^{\left[{ }^{[1]}\right.}$

\section{What policy- and health system-related research has just been completed in SA or is underway?}

The very early infant diagnosis study conducted at Kalafong hospital substantiates the current policy of universal rather than targeted birth HIV testing amongst HIV-exposed newborns. The study demonstrated that using a risk score (threeor four-risks) at a probability of 0.02 and 0.04 , misses $20 \%$ and $24 \%$ of HIV-infected infants, respectively, at birth compared with universal testing. ${ }^{\text {[99] }}$ A field-based evaluation of point-of-care testing for at-birth infant HIV diagnosis, conducted at Rahima Moosa Hospital, highlighted the additional staff needed to make this approach functional; however, the study highlighted the potential child health gains as time to infant ART initiation was reduced. ${ }^{[100]}$ The PHANGISA study will assess the role of viral load and health systems factors in driving peripartum and postpartum MTCT, in the context of PMTCT Option $\mathrm{B}+$, in Ehlanzeni, a rural district in SA (http://www.mrc.ac.za/ intramural-research-units/HealthSystemscurrent-projects).

\section{Research priorities in high HIV-prevalence settings}

CLHIV and HEU children experience unique health challenges that require vigilance and ongoing, long-term surveillance. This need is especially important, given the increasing HEU child population in SA and globally. Care needs to be provided in a non-stigmatising way, and front-line health workers should be aware of the vulnerabilities of these populations.

Several research gaps exist for CLHIV and HEU children in high HIV-prevalence settings (Fig. 1), including understanding mechanisms of preterm birth in WLHIV, to optimise preventive interventions;

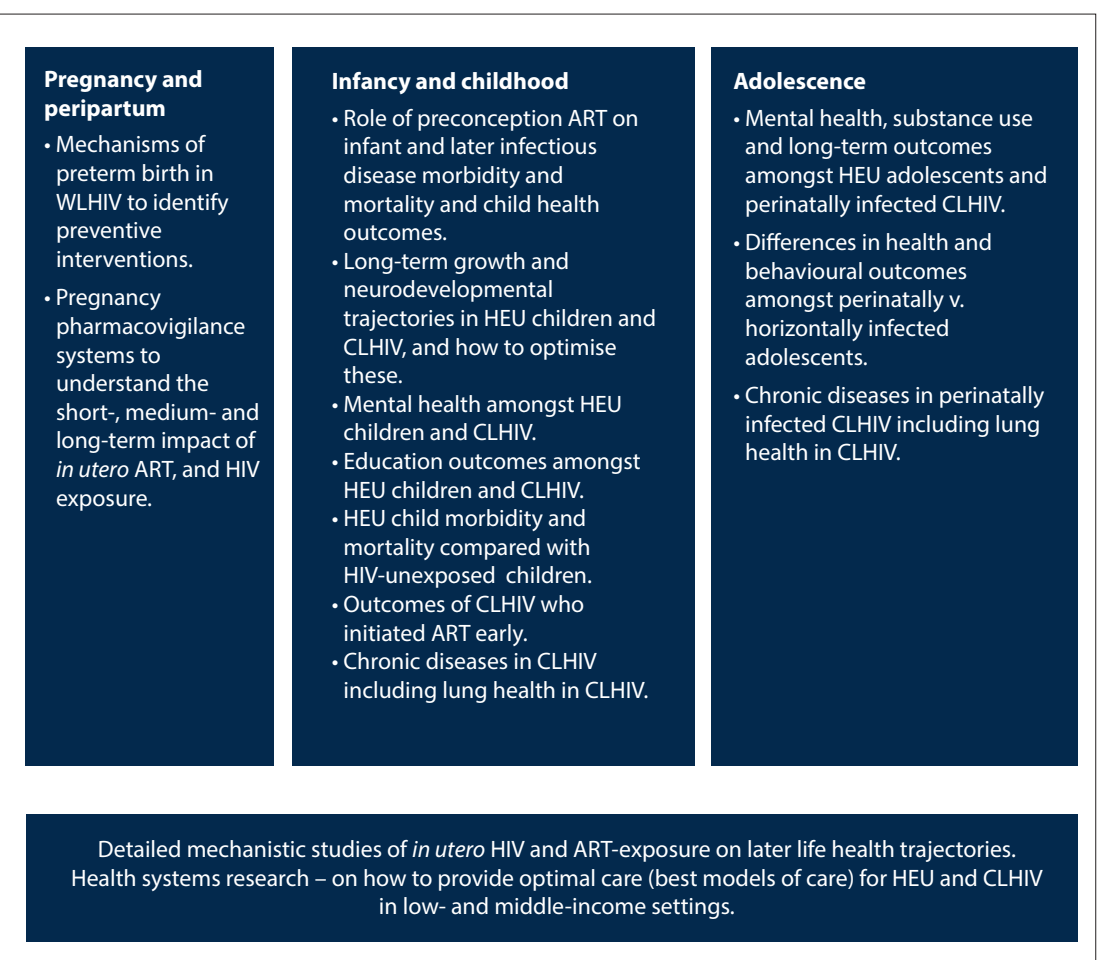

Fig. 1. Research priorities in high HIV-prevalence settings. (WLHIV = women living with HIV; $A R T=$ antiretroviral therapy; $H E U=H I V$-exposed uninfected; $C L H I V=$ children living with HIV.) 
establishing pregnancy pharmacovigilance systems to understand the short-, medium- and long-term impact of in utero ART and HIV exposure; understanding the role of preconception maternal ART on infant infectious morbidity, understanding the role of preconception maternal ART on HEU children's infectious morbidity and long-term growth and neurodevelopmental trajectories in HEU children and CLHIV; understanding mental health outcomes and support required in HEU children and CLHIV through childhood and adolescence; monitoring HEU child morbidity and mortality compared with HIVunexposed children; monitoring outcomes of CLHIV who initiated ART very early in life, sometimes with suboptimal ART regimens due to medication formulation and registration issues; and testing sustainable models of care for HEU children and CLHIV including later reproductive care and support. Lastly, we must not lose sight of the urgent need to reduce maternal HIV prevalence, as this is the most important way to reduce the numbers of CLHIV and HEU.

\section{Conclusion}

More than one in five children in SA are HIV- and ART-exposed in utero; never before has there been exposure to an infectious disease or associated medication use of this magnitude during pregnancy. SA needs to lead the way in better understanding the vulnerabilities of HEU children and CLHIV, to optimise their short- and long-term outcomes.

Acknowledgements. The publication of this paper was supported by the South African Medical Research Council.

Author contributions. Conceptualisation of the paper: A Goga; contribution to paper outline: A Slogrove, V Ramokolo, U Feucht, C Wedderburn; writing specific sections of the paper: A Slogrove, C Wedderburn, V Ramokolo, J Wessels, A Bhana; contributing to technical information in the entire paper, reviewing all sections of the paper, contributing to all sections of the paper, and approving the final version: all authors.

Funding. C Wedderburn was supported by the Wellcome Trust through a Research Training Fellowship (203525/Z/16/Z). V Ramokolo was supported through a post-doctoral fellowship at the SAMRC.

Conflicts of interest. None.

1. Kim M, Ahmed S, Abrams E. Paediatric HIV: Progress on prevention, treatment and cure. Curr Pediatr Rep 2015;3(3):219-229. http://doi.org/10.1007/s40124-015-0087-7

2. Chopra M, Daviaud E, Pattinson R, et al. Saving the lives of South Africa’s mothers, babies, and children: Can 2. Chopra M, Daviaud E, Pattinson R, et al. Saving the lives of South Africass mothers, babies, and children: Can
the health system deliver? Lancet 2009;374(9692):835-846. http://doi.org/10.1016/S0140-6736(09)61123-5 the health system deliver? Lancet 2009;374(9692):835-846. http://doi.org/10.1016/S0140-6736(09)61123-5
3. World Health Organization (WHO). Global guidance on processes and criteria for validation: Elimination of mother-to-child transmission of HIV and syphilis. WHO: Geneva, 2017.

4. The Joint United Nations Programme on HIV/AIDS (UNAIDS). UNAIDS data 2018. UNAIDS: Geneva, 2018.

5. Petra Study Team. Efficacy of three short-course regimens of zidovudine and lamivudine in preventing early and late transmission of HIV-1 from mother to child in Tanzania, South Africa, and Uganda (Petra study): A randomised, double-blind, placebo-controlled trial. Lancet 2002;359(9313):1178-1186. http:// doi.org/10.1016/S0140-6736(02)08214-4

6. Gray G, Urban M, Chersich M, et al. A randomized trial of two post-exposure prophylaxis regimens to 6. Gray G, Urban M, Chersich M, et al. A randomized trial of two post-exposure prophylaxis regimens to
reduce mother-to-child HIV-1 transmission in infants of untreated mothers. AIDS 2005;19(12):1289-1297. reduce mother-to-child HIV-1 transmission in infan
http://doi.org/10.1097/01.aids.0000180100.42770.a7

7. Colvin M, Chopra M, Doherty T, et al. Operational effectiveness of single dose nevirapine in the South African programme to prevent mother-to-child transmission of HIV. Bull World Health Organ 2007;85(6):466-473. https://doi.org/10.2471/blt.06.033639

8. Jackson D, Chopra M, Doherty T, et al Operational effectiveness and 36 week HIV-free survival in the South African programme to prevent mother-to-child transmission of HIV-1. AIDS 2007;21(4):509-516. http://doi.org/10.2471/BLT.06.033639

9. Goga A, Doherty T, Jackson D, et al. Infant feeding practices at routine PMTCT sites, South Africa: Results of a prospective observational study amongst HIV exposed and unexposed infants - birth to 9 months. Int of a prospective observational study amongst HIV exposed and
Breastfeeding J 2012;7:4. https://doi.org/10.1186/1746-4358-7-4

10. Goga A, Dinh T, Jackson D, et al. First population-level effectiveness evaluation of a national programme to prevent HIV transmission from mother to child, South Africa. J Epidemiol Community Health 2015;69(3):240-248. http://doi.org/10.1136/jech-2014-204535

11. Goga A, Dinh T, Jackson D, et al. Population-level effectiveness of maternal antiretroviral treatment initiation before or during the first trimester and infant antiretroviral prophylaxis on early mother-to-child transmission of HIV, South Africa: Implications for eliminating MTCT. J Glob Health 2016;6(2):020405. https://doi.org/10.7189/jogh.6.020405
12. Goga A, Jackson D, Lombard C, et al. Highest risk of mother-to-child transmission of HIV or death in the first 6 months postpartum: Results from 18 month follow-up of an HIV-exposed national cohort the first 6 months postpartum: Results from 18 month follow-up of an HIV-exposed
South Africa. Abstract presented at AIDS 2016 Conference, Durban, South Africa.

13. Sherman G, Haeri Mazanderani A, Barron P, et al. Towards elimination of mother-to-child transmission in South Africa: How best to monitor the PMTCT program. J Glob Health 2017;7(1):010701. http:// doi.org/10.7189/jogh.07.010701

14. National Department of Health (NDoH). National consolidated guidelines for the prevention of mother to child transmission of HIV (PMTCT) and the management of HIV in children, adolescents and adults. NDoH: Pretoria, 2015.

15. World Health Organization (WHO). Global Guidance on Criteria and Processes for Validation Elimination of mother-to-child transmission of HIV and syphilis. WHO: Geneva, 2014.

16. Goga A, Chirinda W, Ngandu N, et al. Closing the gaps to eliminate mother-to-child transmission of HIV (MTCT) in South Africa: Understanding MTCT case rates, factors that hinder the monitorin and attainment of targets, and potential game changers. S Afr Med J 2018;108(3 Suppl 1):S17-S24. and attainment of targets, and potential game ch
http://doi.org/10.7196/SAMJ.2017.v108i3b.12817

17. Goga A, Singh Y, Jackson D, et al. Is elimination of vertical transmission of HIV in high HIV prevalence settings achievable? Brit Med J 2019;364:1687. https://doi.org/10.1136/bmj.11687

18. Goga A, Sherman G, Chirinda W, et al. Eliminating mother-to-child transmission of HIV in South Africa, 2002-2016: Progress, challenges and the last mile plan. In: South African Health Review. Barron P, Paradath A. (eds). Health Systems Trust: Durban, 2017

19. National Department of Health (NDoH). The 2015 National Antenatal HIV and Sypilis Survey Report. NDoH: Pretoria, 2017

20. Wedi CO, Kurtwell S, Hopewell S, et al. Perinatal outcomes associated with maternal HIV infection A systematic review and meta-analysis. Lancet HIV 2016;3(1):e33-e48. http://doi.org/10.1016/S23523018(15)00207-6.

21. Stringer JS, Stoner MC, Kasaro MP, et al. Preconception ART and preterm birth: Real effect or selection bias? Lancet HIV 2017;4(4):e150. https://doi.org/10.1016/S2352-3018(17)30046-2

22. Gibb DM, Kizito H, Russell EC, et al. Pregnancy and infant outcomes among HIV-infected women taking long-term ART with and without Tenofovir in the DART trial. PLoS Med 2012;9(5):e1001217. http://doi: 10.1371/journal.pmed.1001217

23. Powis KM, Kitch D, Ogwu A, et al. Increased risk of preterm delivery among HIV-infected women randomized to protease versus nucleoside reverse transcriptase inhibitor-based HAART during pregnancy. J Infect Dis 2011;204(4):506-514. http:// https://doi.org/10.1093/infdis/jir307

24. Sibiude J, Warszawski J, Tubiana R, et al. Premature delivery in HIV-infected women starting protease inhibitor therapy during pregnancy: Role of the ritonavir boost? Clin Infect Dis 2012;54(9):1348-1360 http:// https://doi.org/10.1093/cid/cis198

25. Malaba T, Phillips T, Le Roux S, et al. Antiretroviral therapy use during pregnancy and adverse birth outcomes in South African women. Int J Epidemiol 2017;46(5):1678-1689. https://doi.org/10.1093/ $\mathrm{ije} / \mathrm{dyx} 136$

26. Zash R, Jacobson D, Diseko M, et al. Comparative safety of dolutegravir-based or efavirenz-based antiretroviral treatment started during pregnancy in Botswana: An observational study. Lancet Glob Health 2018;6(7):e804-e810. https://doi.org/10.1016/S2214-109X(18)30218-3

27. Uthman O, Nachega J, Anderson J, et al. Timing of initiation of antiretroviral therapy and adverse pregnancy outcomes: A systematic review and meta-analysis. Lancet HIV 2016;4(1):e21-e30. https:// doi.org/10.1016/S2352-3018(1016)30195-30193

28. Zash R, Makhema J, Shapiro R. Neural tube defects with dolutegravir treatment from the time of conception. N Engl J Med 2018;379(10):979-981. https://doi.org/10.1056/NEJMc1807653

29. Mehta U, Kalk E, Boulle A, et al. Pharmacovigilance: public health priority for South Africa. In: South African Health Review. Barron P, Paradath A. (eds). Health Systems Trust: Durban, 2017.

30. Scrimshaw NS. Historical concepts of interactions, synergism and antagonism between nutrition and infection. J Nutr 2003;133(1):316S-321S. http://doi.org/10.1093/jn/133.1.316S

31. Bourke CD, Berkley JA, Prendergast AJ. Immune dysfunction as a cause and consequence of malnutrition. Trends Immunol 2016;37(6):386-398. http://doi.org/10.1016/j.it.2016.04.003

32. Ramokolo V, Lombard C, Fadnes LT, et al. HIV infection, viral load, low birth weight, and nevirapine are independent influences on growth velocity in HIV-exposed South African infants. J Nutr 2014;144(1):42-48. https://doi.org/10.3945/jn.113.178616

33. Isanaka S, Duggan C, Fawzi WW. Patterns of postnatal growth in HIV-infected and HIV-exposed Isanaka S, Duggan C, Fawzi WW. Patterns of postnatal growth in HIV-infected and
children. Nutr Rev 2009;67(6):343-359. http://doi.org/10.1111/j.1753-4887.2009.00207.x

34. Le Roux S, Abrams E, Donald K, Brittain K, Phillips T, Nguyen K. Growth trajectories of breastfed HIV-exposed uninfected and HIV-unexposed children under conditions of universal maternal antiretroviral therapy: A prospective study. Lancet Child Adolesc Health 2019;3(4):234-244. https:// doi.org/10.1016/S2352-4642(19)30007-0

35. Le Roux SM, Abrams EJ, Donald KA, et al. Growth trajectories of breastfed HIV-exposed uninfected and HIV-unexposed children under conditions of universal maternal antiretroviral therapy: prospective study. Lancet Child Adolesc Health 2019;3(4):234-244. https://doi.org/10.1016/S23524642(19)30007-0

36. Malaba TR, Phillips T, Le Roux S, et al. Antiretroviral therapy use during pregnancy and adverse birth outcomes in South African women. Int J Epidemiol 2017;46(5):1678-1689. https://doi.org/10.1093/ ije/dyx 136

37. Ramokolo V, Goga AE, Lombard C, et al. In utero ART exposure and birth and early growth outcomes among HIV-exposed uninfected infants attending immunization services: Results from national PMTCT surveillance, South Africa. Open Forum Infect Dis 2017;4(4):ofx187. https:///doi.org/10.1093/ PMTCT sur
ofid/of $x 187$

38. Wedi CO, Kirtley S, Hopewell S, et al. Perinatal outcomes associated with maternal HIV infection: A systematic review and meta-analysis. Lancet HIV 2016;3(1):e33-e48. https://doi.org/10.1016/S2352 3018(15)00207-6

39. Evans C, Humphrey JH, Ntozini R, et al. HIV-exposed uninfected infants in Zimbabwe: Insight into health outcomes in the pre-antiretroviral therapy era. Front Immunol 2016;7:190. http://do org/10.3389/fimmu.2016.00190

40. Powis KM, Smeaton L, Hughes MD, et al. In-utero triple antiretroviral exposure associated with decreased growth among HIV-exposed uninfected infants in Botswana. AIDS 2016;30(2):211-220. http://doi.org/10.1097/QAD.0000000000000895

41. Abubakar A, Van Baar A, Van de Vijver FJ, et al. Paediatric HIV and neurodevelopment in sub-Saharan Africa: A systematic review. Trop Med Int Health 2008;13(7):880-887. https://doi.org/10.1111/j.13653156.2008.02079.x

42. Govender R, Eley B, Walker K, et al. Neurologic and neurobehavioral sequelae in children with human immunodeficiency virus (HIV-1) infection. J Child Neurol 2011;26(11):1355-1364. http://doi. org/10.1177/0883073811405203.

43. Wilmshurst JM, Hammond CK, Donald K, et al. NeuroAIDS in children. In: Handbook of Clinical Neurology. Vol 152. Brew BJ (ed). Elsevier: Netherlands, 2018:99-116.

44. Hoare J, Ransford GL, Phillips N, et al. Systematic review of neuroimaging studies in vertically transmitted HIV-positive children and adolescents. Metab Brain Dis 2014; 29(2):221-229. https://doi org/10.1007/s11011-013-9456-5

45. Musielak KA, Fine JG. An updated systematic review of neuroimaging studies of children and adolescents with perinatally acquired HIV. J Pediatr Neuropsych 2015;2(1-2):34-49. https://doi org/10.1007/s40817-015-0009-1 
46. Whitehead N, Potterton J, Coovadia A. The neurodevelopment of HIV-infected infants on HAART compared to HIV-exposed but uninfected infants. AIDS Care 2014;26(4):497-504. https://doi.org/10 1080/09540121.2013.841828

47. Le Doare K, Bland R, Newell ML. Neurodevelopment in children born to HIV-infected mother by infection and treatment status. Pediatrics 2012;130(5):el326-e1344. https://doi.org/10.1542/ peds.2012-0405

48. Boivin MJ, Barlow-Mosha L, Chernoff MC, et al. Neuropsychological performance in African children with HIV enrolled in a multisite antiretroviral clinical trial. AIDS 2018;32(2):189-204. https://do org/10.1097/QAD.0000000000001683

9. McHenry MS, McAteer CI, Oyungu E, et al. Neurodevelopment in young children born to HIV infected mothers: A meta-analysis. Pediatrics 2018;141(2):e20172888. http://doi.org/10.1097/ QAD. 0000000000001683

50. Sherr L, Croome N, Parra CK, et al. A systematic review of psychological functioning of children exposed to HIV: using evidence to plan for tomorrow's HIV needs. AIDS Behav 2014;18(11):20592074. https://doi.org/10.1007/s10461-014-0747-6

51. Le Roux SM, Donald KA, Brittain K, et al. Neurodevelopment of breastfed HIV-exposed uninfected and HIV-unexposed children in South Africa. AIDS 2018;32(13):1781-1791. http://doi.org/10.1097 QAD. 0000000000001872

52. Nicholson L, Chisenga M, Siame J, et al. Growth and health outcomes at school age in HIV-exposed, uninfected Zambian children: Follow-up of two cohorts studied in infancy. BMC Pediatrics 2015;15:66. http://doi.org/10.1186/s12887-015-0386-8

53. Chaudhury S, Williams PL, Mayondi GK, et al. Neurodevelopment of HIV-exposed and HIVunexposed uninfected children at 24 months. Pediatrics 2017;140(4):e20170988. http://doi. unexposed uninfected childe

54. Springer PE, Slogrove AL, Laughton B, et al. Neurodevelopmental outcome of HIV-exposed but Springer PE, Slogrove AL, Laughton B, et al. Neurodevelopmental outcome of HIV-exposed but
uninfected infants in the Mother and Infants Health Study, Cape Town, South Africa. Trop Med Int

55. Rice ML, Russell JS, Frederick T, et al. Risk for speech and language impairments in preschool age HIV-exposed uninfected children with in utero combination antiretroviral exposure. Pediatr Infect Dis J 2018;37(7):678-685. http://doi.org/10.1097/INF.000000000000187

56. Buchacz K, Rogol A, Lindsey J, et al. Delayed onset of pubertal development in children and adolescents with perinatally acquired HIV infection. J Acquir Imune Defic Syndr 2003;33(1):56-65 http://doi.org/10.1097/00126334-200305010-00009

57. Brackis-Cott E, Kang E, Dolezal C, et al. Brief report: Language ability and school functioning of youth perinatally infected with HIV. J Pediatr Health Care 2009;23(3):158-164. http://doi.org/10.1016/j. pedhc.2008.02.005

58. Nozyce M, Lee S, Wiznia A, et al. A behavioral and cognitive profile of clinically stable HIV-infected children. Pediatrics 2006;1173(3):763-770. http://doi.org/10.1542/peds.2005-0451

59. Donenberg G, Pao M. Youths and HIV/AIDS: Psychiatry's role in a changing epidemic. J Am Acad Child Adolec Psychiatry 2005;44(8):728-747. https://doi.org/10.1097/01.chi.0000166381.68392.02

60. Nassen R, Donald K, Walker K, et al. Management of mental health disorders and central nervous Nassen R, Donald K, Walker K, et al. Management of mental health disorders and central
system sequelae in HIV-positive children and adolescents. S Afr J HIV Med 2014;15(3):81-96.

61. Mellins C, Tassiopoulos K, Malee K, et al. Behavioral health risks in perinatally HIV-exposed youth: Co-occurrence of sexual and drug use behavior, mental health problems, and nonadherence youth: Co-occurrence of sexual and drug use behavior, mental health problems, and nonadherence
to antiretroviral treatment. AIDS Patient Care STDS 2011;25(7):413-422. https://doi.org/10.1089/ to antiretrovir

62. Gadow K, Angelidou K, Chernoff M, et al. Longitudinal study of emerging mental health concerns in youth perinatally infected with HIV and peer comparisons. J Dev Behav Pediatr 2012;33(6):456-468. http://doi.org/10.1097/DBP.0b013e31825b8482

63. Mellins C, Malee K. Understanding the mental health of youth living with perinatal HIV infection: Lessons learned and current challenges. J Int AIDS Soc 2013;16:18593. https://doi.org/10.7448/ IAS.7416.7441.18593

64. Sherr L, Cluver LD, Toska E, et al. Differing psychological vulnerabilities among behaviourally and perinatally HIV infected adolescents in South Africa - implications for targeted health service provision. AIDS Care 2018;30(Suppl 2):92-101. http://doi.org/10.1080/09540121.2018.1476664

65. Brittain K, Myer L, Phillips N, et al. Behavioural health risks during early adolescence among perinatally HIV-infected South African adolescents and same-age, HIV-uninfected peers. AIDS Care 2019;31(1):131-140. https://doi.org/10.1080/09540121.2018.1533233

66. Heymann J, Kidman R. HIV/AIDS, declining family resources and the community safety net. AIDS 2009;21(Suppl 1):34-42. https://doi.org/10.1080\%2F09540120902927593

67. Newell ML, Coovadia H, Cortina-Borja M, et al. Mortality of infected and uninfected infants born to HIV-infected mothers in Africa: A pooled analysis. Lancet 2004;364(9441):1236-1243. https://doi. to HIV-infected mothers in Africa: $\mathrm{A}$ -
org/10.1016/S0140-6736(04) 17140-7

68. Frigati L, Archary M, Rabie H, et al. Priorities for decreasing morbidity and mortality in children with advanced HIV disease. Clin Infect Dis 2018;66(Suppl 2):S147-S151. https://doi.org/10.1093/cid/ciy013

69. Technau K-G, Strehlau R, Patel F, et al. 12-month outcomes of HIV-infected infants identified a birth at one maternity site in Johannesburg, South Africa: An observational cohort study. Lancet HIY 2018;5(12):e706-e714. https://doi.org/10.1016/\$2352-3018(18)30251-0

70. Taron-Brocard C, Le Chenadec J, Faye A, et al. Increased risk of serious bacterial infections due to maternal immunosuppression in HIV-exposed uninfected infants in a European country. Clin Infect Dis 2014;59(9):1332-1345. https://doi.org/10.1093/cid/ciu586

1. Arikawa S, Rollins N, Newell ML, et al. Mortality risk and associated factors in HIV-exposed, uninfected children. Trop Med Int Health 2016;21(6):720-734. http://doi.org/10.1111/tmi.12695

72. Brennan AT, Bonawitz R, Gill CJ, et al. A meta-analysis assessing all-cause mortality in HIV-exposed uninfected compared with HIV-unexposed uninfected infants and children. AIDS 2016;30(15):23512360. http://doi.org/10.1097/QAD.0000000000001211

73. Slogrove A, Johnson LFP, Powis KM. Population-level mortality associated with HIV exposure in HIV-uninfected Infants in Botswana and South Africa: A model-based evaluation. J Trop Pediatr 2018;65(4):373-379. https://doi.org/10.1093/tropej/fmy064
74. Yeganeh N, Watts DH, Xu J, et al. Infectious morbidity, mortality and nutrition in HIV-exposed, uninfected, formula-fed infants: Results from the HPTN 040/PACTG 1043 Trial. Pediatr Infect Dis J 2018:37(12):1271-1278. https://doi.org/10.1097/INF.0000000000002082

75. Von Mollendorf C, von Gottberg A, Tempia S, et al. Increased risk for and mortality from invasive pneumococcal disease in HIV-exposed but uninfected infants aged $<1$ year in South Africa, 2009 2013. Clin Infect Dis 2015;60(9):1346-1356. https://doi.org/10.1093/cid/civ059

76. Cohen C, Moyes J, Tempia S, et al. Epidemiology of acute lower respiratory tract infection in HIVexposed uninfected infants. Pediatrics 2016;137(4):e20153272. https://doi.org/10.1093/cid/civ059

77. Verani JR, Groome MJ, Zar HJ, et al. Risk factors for presumed bacterial pneumonia among HIVuninfected children hospitalized in Soweto, South Africa. Pediatr Infect Dis J 2016;35(11):11691174. http://doi.org/10.1097/INF.0000000000001264

78. Le Roux DM, Myer L, Nicol MP, et al. Incidence and severity of childhood pneumonia in the first year of life in a South African birth cohort: The Drakenstein Child Health Study. Lancet Glob Health 2015;3(2):e95-e103. https://doi.org/10.1016/S2214-109X(14)70360-2

79. Lockman S, Hughes M, Powis K, et al. Effect of co-trimoxazole on mortality in HIV-exposed but uninfected children in Botswana (the Mpepu Study): A double-blind, randomised, placebocontrolled trial. Lancet Glo Health 2017;5(5):e491-e500. https://doi.org/10.1016/S2214109X(17)30143-2

80. Goetghebuer T, Smolen KK, Adler C, et al. Initiation of antiretroviral therapy before pregnancy reduces the risk of infection-related hospitalization in human immunodeficiency virus-exposed uninfected infants born in a high-income country. Clin Infect Dis 2019;68(7):1193-1203. https:// doi.org/10.1093/cid/ciy673

81. Slogrove A, Reikie B, Naidoo S, et al. HIV-exposed uninfected infants are at increased risk for severe infections in the first year of life. J Trop Pediatr 2012;58(6):505-508. http://doi.org/10.1093/tropej/ inso19

82. Slogrove AL, Goetghebuer T, Cotton MF, et al. Pattern of infectious morbidity in HIVexposed uninfected infants and children. Front Immunol 2016;7:164. http://doi.org/10.3389/ fimmu.2016.00164

83. Le Roux DM, Nicol MP, Myer L, et al. Lower respiratory tract infections in children in a wellvaccinated South African birth cohort: Spectrum of disease and risk factors. Clin Infect Dis 2019:ciz017 (in press). https://doi.org/10.1093/cid/ciz017

84. Rabie H, Goussard P. Tuberculosis and pneumonia in HIV-infected children: An overview. Pneumonia 2016;8:19. https://doi.org/10.1186/s41479-016-0021-y

85. Venturini E, Turkova A, Chiappini E, et al. Tuberculosis and HIV co-infection in children. BMC Infect Dis 2014;14(Suppl 1):S5. https://doi.org/10.1186/1471-2334-1114-S1181-S1185

86. Hesseling A, Cotton M, Jennings T, et al. High incidence of tuberculosis among HIV-infected infants: Evidence from a South African population-based study highlights the need for improved tuberculosis control strategies. Clin Infect Dis 2009;48(1):108-114. https://doi.org/110.1086/595012

87. Walters $\mathrm{E}$, Cotton $\mathrm{M}$, Rabie H, et al. Clinical presentation and outcome of tuberculosis in human immunodeficiency virus infected children on anti-retroviral therapy. BMC Pediatrics 2008;8(1). https://doi.org/10.1186/1471-2431-1188-1181

88. Martinson N, Moultrie H, van Niekerk R, et al. HAART and risk of tuberculosis in HIV-infected South African children: A multi-site retrospective cohort. Int J Tuberc Lung Dis 2009;13(7):862-867,

89. Dangor Z, Izu A, Hillier K, et al. Impact of the antiretroviral treatment program on the burden of hospitalization for culture-confirmed tuberculosis in South African children: A time-series analysis. Pediatr Infect Dis J 2013;32(9):972-977. https://doi.org/10.1097/INF.0b013e31828d9aa4

90. Fairlie L, Beylis NC, Reubenson G, et al. High prevalence of childhood multi-drug resistant tuberculosis in Johannesburg, South Africa: A cross sectional study. BMC Infect Dis 2011;11(1):28. https://doi.org/10.1186/1471-2334-11-28

91. Hicks R, Padayatchi N, Shah N, et al. Malnutrition associated with unfavorable outcome and death among South African MDR-TB and HIV co-infected children. Int J Tuberc Lung Dis 2014;18(9):1074-1083: https://doi.org/1010.5588/ijtld.1014.0231.

92. Seddon JA, Hesseling AC, Willemse M, et al. Culture-confirmed multidrug-resistant tuberculosis in children: Clinical features, treatment, and outcome. Clin Infect Dis 2011;54(2):157-166. https:// doi.org/10.1093/cid/cir772

93. Kitchin O, Masekela R, Moodley T, et al. Outcome of HIV exposed and infected children admitted to a pediatric intensive care unit for respiratory failure. Pediatr Crit Care Med 2012;13(5):516-519. http://doi.org/10.1097/PCC.0b013e31824ea143.

94. Ferrand RA, Desai SR, Hopkins C, et al. Chronic lung disease in adolescents with delayed diagnosis of vertically-acquired HIV infection. Clin Infect Dis 2012;55(1):142-152. https://doi.org/10.1093/ cid/cis 271

95. Weber $\mathrm{H}$, Gie R, Wills $\mathrm{K}$, Cotton M. Clinical features and lung function in HIV-infected children with chronic lung disease. S Afr J Child Health 2015;9(3):72-75. http://doi.org/10.7196/SAJCH.7940

6. Calligaro G, Gray D. Lung function abnormalities in HIV-infected adults and children. Respirology 2015;20(1):24-32. https://doi.org/10.1111/resp.12385

97. Solarin I, Black V. 'They told me to come back': Women's antenatal care booking experience in innercity Johannesburg. Matern Child Health J 2013;17(2):359-367. https://doi.org/10.1007/s10995-012$1019-6$

98. Sherry K. Disability and rehabilitation: Essential considerations for equitable, accessible and poverty-reducing health care in South Africa. In: South African Health Review. Padarath A, King J, English R. (eds). Health Systems Trust: Durban, 2015.

99. du Plessis N, Muller C, Avenant T, et al. An early-infant HIV-risk score for targeted HIV testing at Dirth: The VEID study, South Africa. Paediatrics 2019;143(6).

100. Technau K-G, Kuhn L, Coovadia A, et al. Xpert HIV-1 point-of-care test for neonatal diagnosis of HIV in the birth testing programme of a maternity hospital: A field evaluation study. Lancet HIV 2017;4(10):e442-e448. https://doi.org/10.1016/S2352-3018(17)30097-8 